\title{
Towards an Innovative Validation-Driven Approach to Lean Product Development
}

\author{
Osama Al-Baik \\ Dept. of Electr. \& Comput. Eng. \\ University of Alberta \\ albaik@ualberta.ca
}

\author{
James Miller \\ Dept. of Electr. \& Comput. Eng. \\ University of Alberta \\ albaik@ualberta.ca
}

\author{
Daniel R Greening \\ Senex Rex \\ dan@senexrex.com
}

\begin{abstract}
Many software products contain unnecessary functionality. Industrial reports show $45 \%$ of the features in analyzed software products are never used. Software-centric organizations have been trying to sustain their competitive advantage by re-defining their product development strategy. Recent attempts to re-strategize the product development process tend towards customer-centric approaches. We propose a validation-driven model based around proven lean principles, agile methods, and value-driven design. To increase the model's likelihood of success, the study discusses suitable guidelines and deployment protocols that have been evolved in industrial settings.
\end{abstract}

\section{Introduction}

The software industry faces a major problem - how to define features that customers want to use and are willing to pay for. Software-centric organizations have traditionally concentrated on approaches such as model-driven architecture (MDA) [21], which provides methods to define product specifications. Recent development, however, especially in industrial settings, have started to challenge these ideas and this proposal seeks to build upon these fledgling ideas [1, 24, 25].

There is an underlying need to understand and clearly define the customers' problems that a software product is intended to solve. The hypothesis is that value to the customer should be the principle driver when designing a software product.

This study seeks to represent a model that defines value from "cradle to grave". Establishing a value proposition should start the deployment process and essentially end it. Hence, the objectives of this research article have been formulated as follows:
(1) Characterize models that describe, and only describe, the essence of a software product idea;

(2) Guide the refinement and evolution of these models; and

(3) Develop a deployment protocol to these models as mechanisms to achieve exploring potential products;

The proposed validation model has been established around the Lean Startup concepts as defined by Eric Ries [25]. The model concentrates on the early stages of the product lifecycle. The development, deployment, and refinement of the proposed validation-driven model started in 2014. The validation model and its associated deployment protocol have matured in testing with an industrial partner;

During the past 15 months, a total of 38 participants have been actively involved in developing and refining the proposed model, which has been inuse with the industrial partner for several months. The scope of this study, however, is limited to the evolution of the model, its components, and deployment protocol. It excludes the evaluation of the model as the model has not been used long-enough to produce reliable results.

The reminder of this paper is organized as follows. Section 2 discusses the problem statement. Section 3 describes the proposed validation model characteristics and components, and describes, research scope and context. Section 4 describes the chronological phases of the implementation protocol. Section 5 summarizes the methodology. Section 6 discusses how the model integrates lean principles, agile methods, and valuedriven design. Section 7 provides insight into related work in this area, while section 8 summarizes the conclusions of this study.

Acknowledgment. This research was funded by the industrial partner and NSERC grant CRDJ 4683642014. The authors gratefully acknowledge the support provided to carry this research to-date. 


\section{Problem Statement}

Many software systems contain unnecessary functionality. Johnson [15] reports that $45 \%$ of the features in analyzed systems were never used. Juergens et al. [10], on the usage of an industrial business information systems, shows that $28 \%$ of their features were unnecessary. While, the accuracy of the quoted numbers can be debated, they demonstrate that capturing the real requirements for a product is a poorly understood process.

This is especially true when constructing for the mass marketplace. Currently, in agile development, user stories approach [13] is the leading industrial software development approach. It overcomes major limitations and weaknesses of predecessor approaches, such as rigidity, hard to estimate cost, and limited user input; however, it suffers from other critical issues:

A proxy for the customer. Normally, few people, often a single individual (e.g. a Product Manager), represents the user community. This small group can misrepresent the opinions of real user community.

The proxy is often embedded with the development team. While this has positive advantages, the proxy often becomes biased to accept requirements presented by their teammates.

Boolean Acceptance Tests. Acceptance tests are often simple yes / no decisions. Can complex ideas, such as usability, really be devolved into a number of simple questions as in acceptance testing frameworks?

Lack of big picture. User stories concentrate on a divide and conquer strategy of production. Teams are often asked to concentrate on the current sprint. Hence, consideration of the big picture is very infrequent.

Functional Details only. Since user stories drive the work plan and the person-allocation, they tend to concentrate on work packages - building code. Details on other aspects of the project are unrepresented in these stories. In other words, user stories lack considering "support functions" of product development, such as sales and marketing functions.

In order to overcome these issues, better understand the customers, and to stay competitive in the rapidly changing software-centric market, many organizations have been looking into re-strategizing the product development processes around customer-centric approaches; evidence for this can be found in [1, 24].

The key is to develop a working definition of value. The unsystematic and unorganized addition of new product ideas to development backlogs, without having a well-defined approach to validate the value it provides to the customer base, will ultimately result in creating unnecessary features which in turn, will lead to wasteful products with no clear requirements, and no clear customer-centric "value add" definition.

\section{The Validation Model Evolution}

Software Engineering approaches have traditionally concentrated on ideas such as model-driven architecture (MDA) to stimulate specifications and represent them in conceptual models; however, these approaches tend to struggle to accommodate the high degree of uncertainty associated with bringing new ideas to fruition, especially in products aimed at mass markets.

MDA, however, has good points - the idea that a product needs to be more than its code base is believed to be correct. MDA, and other traditional models focus on providing artefacts principally for verification, whereas in this article, we argue that these models need to be explicit for validation purposes.

\subsection{Study Scope}

A validation-driven model has evolved and work has started to develop, deploy, and refine the proposed model early in 2014, however, a formal research design was launched in October 2014. Table 1 shows a Gantt chart of the research project.

As illustrated in Table 1, the investigators planned to report their findings in two distinct reports. The first report on one hand, aims to define and illuminate the different components of the validation-driven model, and to establish guidelines that inform the implementation protocol.

The second report in the other hand reports the impact of the validation model on the overall performance of the organization. The results of using the model cannot be claimed to be reliable before using the model for an extended period. Hence, the results of implementing the model will be informed in a separate report, and thus are excluded from this study.

\subsection{Study Context}

This report concentrates on the evolution of the model and its deployment protocol. Hence, it is important to describe the environment and the context where the model has evolved. To maintain the confidentially, the investigators have used the arbitrary name "IndPar" for our industrial partner.

IndPar is a privately held small-sized software development organization with 32 developers, designers, and architects. The management team is composed of a CEO, Manager of HR and Accounting, a Product Development Manager, and 3 mid-level managers dedicated for sales and marketing. 
IndPar provides a full spectrum of information technology services with particular emphasis on Enterprise Content Management (ECM) and Business Intelligence". For the past 3 years, IndPar has been working on developing a software product to increase the value of existing enterprise applications through enhanced functionality and in-depth intelligence.

The product has been designed as a core platform with several plug-and-play modules. The product's core concept is that it unobtrusively adds markups to existing web applications without requiring costly modifications and releases. Once activated, it "injects" new features that enhance, extend, and connect web applications regardless of their underlying technology.

A major challenge for IndPar was dealing with the numerous enhancements the development team suggested to the ECM and BI capabilities. This was expected as the team has knowledge and experience in these product areas.

Another major challenge was that most of the development team's ideas were technical. New ideas were added "randomly" by team members to the development backlog with no clear customer-centric value. To overcome these challenges, the investigators suggested a novel approach that is based around lean concepts, agile methods, and value-driven design. That is, a validation-driven approach.

\subsection{Validation Model Characteristics}

Validation models are vehicles for exploration and learning. Peter Senge [28] argues that the most sustainable competitive advantage, which any organization should strive to maintain, is to learn faster than competitors. Learning about the customer base to anticipate their needs not only keeps current customers happy, but also helps generate unique product ideas.

Good product ideas emerge from experiments where feature ideas are exposed to a large customer base. A project normally starts with a single concept, framed as a validation model. This model is minimally realised, possibly including realisations that are not code based, to enable customer based experiments. The results from experiments assist in evaluating current ideas and to generate further ideas, which are then themselves subjects for further experimentation.

Evidence-based practices suggest that these experiments start generating evidence about which ideas will likely attract the customer usage and which will not. To allow these experiments to be designed and deployed in the most effective way, the proposed validation model has been built around combining Lean Startup [25], Agile, and Value-Driven design concepts. The proposed validation model possesses the following characteristics:
Support multiple concurrent experiments. The goal is to experiment with as many product ideas as possible, in the shortest time. Several concurrent experiments allow the development team to learn at the maximum pace. Concurrent experiments also support the idea of a portfolio of experiments.

Support experiments from different perspectives. The experiments in a portfolio should ideally be orthogonal. For example, experiments designed by product domain experts (too much background) and experiments designed by experts from a distant field (too little background), experiments central to the domain and experiments oblique to the domain, low-risk, low-reward (evolution) and high-risk, high-reward (revolution) experiments. These orthogonal viewpoints provide contradiction and hence provide an ideal basis for reflection and evaluation.

Support Coherence Examination. Every possible experiment is not worth running; hence the model must contain sufficient information for stakeholders to refute obviously defective ideas, this should help to direct the efforts on experimenting the ideas with higher likelihood to attract and ultimately add value to the customer base.

Support safe-to-fail examination. Experiments need to be low cost - to allow multiple experiments. At this point, evolutionary experiments should be the dominant approach. Once, the idea proves to be appealing to the customer base, more revolutionary experiments may be executed. In addition to the tangible costs, the team should also consider intangible costs such as negative impact on customers.

Iterative and Quick. These models are learning models; many models will be produced as we learn about the domain and the customers. Hence, these models need to be quick to produce. The details about the product must be contained within the instruments to be used during the experiments.

Visualization. Kanban boards have demonstrated the success of stakeholders being able to see and understand the status of a product or production run [2]. Validation models must possess the same quality.

\subsection{Validation Model Components}

According to Love and Back [18], models are not always valid, and there is always a need to consider factors that will enhance the creation of benefits and minimization of risks and challenges. Thus, the investigators have worked for an extended period to develop a validation-driven model that has gone through multi-refinement stages to maximize the benefits and minimize challenges. The model is illustrated in Figure 1 below. 


\begin{tabular}{|c|c|c|c|c|}
\hline \multirow{4}{*}{ 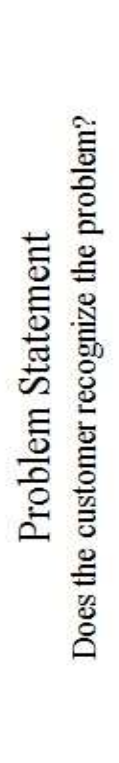 } & $\begin{array}{l}\text { Uniqueness } \\
\text { How to prevent similar product offerings? }\end{array}$ & \multirow{3}{*}{ 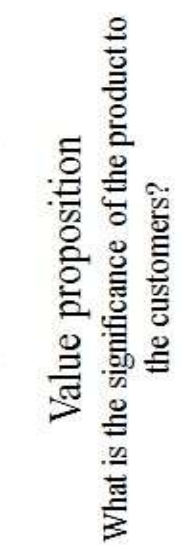 } & \multirow{3}{*}{ 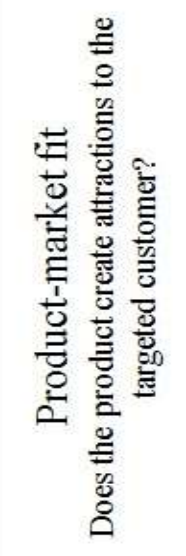 } & \multirow{4}{*}{ 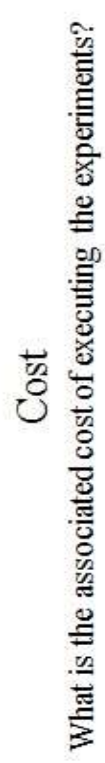 } \\
\hline & $\begin{array}{l}\text { Solution Formulation } \\
\text { What is minimum feature set needed to start } \\
\text { visualizing the solution? }\end{array}$ & & & \\
\hline & $\begin{array}{l}\text { Customer Segmentation } \\
\text { Whom is impacted by the problem? What } \\
\text { are the targeted segments? Vertical / } \\
\text { Horizontal Segments? }\end{array}$ & & & \\
\hline & $\begin{array}{l}\text { Communications \& Interactions } \\
\text { How do we interact with the customers? }\end{array}$ & \multicolumn{2}{|c|}{$\begin{array}{l}\text { Key Measures } \\
\text { What are the key metrics to } \\
\text { assess solution viability? }\end{array}$} & \\
\hline \multicolumn{5}{|c|}{$\begin{array}{l}\text { Revenue Model } \\
\text { What are the revenue streams? }\end{array}$} \\
\hline
\end{tabular}

Figure 1. Proposed Validation-Driven Components

The validation model components in Figure 1 are presented to show the relationships between them, topdown and left-right. For example, to determine the appropriate Revenue Model, the other components laying above it should be considered, while to determine the most informative Key Measures, the metrics should consider Value Proposition, ProductMarket fit, and Communication and Interactions, which implicitly considers Customer Segmentation, and so on. Following is a detailed description of the identified core model componenets:

Problem statement. The addition of any new module, or the addition of a new feature to an existing module, must be directly related to adding value to the customer. Hence, the need has become apparent to understand and define the customers' problems that the product is intended to solve. In order to capture the customers' perceptions about the problem, the problem definition should be expressed based upon feedback that is presented by the voice of the customer [4]. This is the first validation checkpoint, making sure that the customers recognize the problem that the product is intended to solve.

Customer segmentation. Identifying customer segments is as important as defining the problem. In fact, the customer segments should be identified as early as the problem definition stage, as the customers will be the primary determinant to whether the problem is worth solving. The segmentation should be based upon the identification of customers who have similar characteristics and behave in comparable ways segment homogeneity.

The segmentations might have a verticalsegmentation [22] where a product is targeting problems in a particular industry or profession, or a horizontal-segmentation [22] where a product is targeting a specific problem across various industries. Regardless of the segmentation technique, the segmentation will help in defining different challenges, and a possible way to come up with information essential in developing solutions.

Communications and Interactions. How do we interact with the customers? Building a path to the customers is an important step to realise the proposed model [25]. As a matter of fact, the entire validationdriven model is based upon evidence gathered from customers. The use of mass communications guarantees reaching a large customers base, this will later help in assessing how appealing the solution is by measuring the number of attracted, activated, and retained customers [19].

Approaching customers can be very expensive; however, we need to keep the cost as low as possible to support the model characteristic "Support safe-to-fail examination" to allow multiple experiments. Reaching out to customers might be realised using various means: Social media, blogs, webinars, tradeshows, conferences, workshops, search engine marketing (SEM), and others. Following the same validation model, the interaction model would be designed to test 
multiple low-cost channels to reach a larger customer base. The interaction model should be based around the product, the customer segment and the experiment itself.

Solution formulation. When the problem is understood and proves to worth solving by the identified customer segments, the solution should be formulated based upon the minimum feature set needed to start visualizing the solution and validate that it will solve the identified problem. The proposed technique to commercialize the solution, and transform it into a product, is Minimum Viable Product (MVP) [25]. MVP helps in visualizing the solution that provides just enough features to be tested by the customers. MVP is validated by early adopters, who are more interested in the product and are more willing to provide feedback.

The focus, at this stage, is on the problems of the early adopters. This helps in designing the experiments and specifying the solution. After each experiment, analysis is conducted to understand how new customers were attracted? And what were the strategies to retain them? This reflection assists in identifying what worked and why, what did not work and why, and how to emphasize these strategies to attract more customers of similar profiles in the future.

Costs. The costs of developing the product, especially during the early stages, cannot be accurately estimated. Thus, it is important to estimate the costs of each MVP and experiment from the bottom-up, the cost should be based around the operational cost that are incurred while acquiring the potential customers, the cost of developing MVPs and constructing other experimental material.

Activity-based costing [31] is a methodology that can be used to relate the cost of each activity with a specific resource based on the actual consumption of the resource or the resource's time. The activity-based costing allows the assignment of more indirect costs and overheads into direct costs [31]. The use of the classic bootstrapping estimation [16] is another technique that makes it possible for the costs to be identified based on the investment that is made on all development stages. Interactions with the customers will enable efforts towards development to be more focused, which in turn will enhance cost reduction to attain product competitive advantages.

Value proposition. Value proposition is the significance of the product to the customers in terms of creating a difference and adding value. The value proposition should be designed to fit a specific customer segment; and subsequently communicated through the interaction channels to attract potential customers. The product may have several value propositions targeting different customer segments
[26]. For one customer segment, the value proposition might be emphasizing how it would alleviate the customers' principal-problem of undesired cost, business process inefficiencies, or risks. For another customer segment, the value proposition might be formulated around the benefits realization of strategic alignment or other positive outcomes.

Product-market Fit. The mapping of the value proposition features to the characteristics of the customer segment profiles creates a problem-solution fit [23]. Once the problem-solution fit has been validated with the customer base, the product-market fit [23] can then be determined by evaluating whether the product creates attractions to the targeted customer segments. At this stage, if and only if the product has proven successful in attracting the customer base's attention, then it would be considered for further exploration and development.

Revenue Model. Once the product-market fit is achieved, the revenue model can be established. Revenue can be realised using various streams, including: direct versus indirect, reoccurring revenue versus one-time revenue, and leasing versus licensing. Similar to the value proposition, the revenue may have several models that each designed to fit specific customer segment [3].

Key Measures. Measuring the key performance indicators of the product is an integral part of the proposed model. The proposed measuring metric are the "Pirate Metrics - AARRR" [20]; it measures 5 elements:

(a) Acquisition, which measures the customers' interest in the newly proposed product or feature;

(b) Activation, which measures the rate of acquired potential customers that took an action towards exploring the product or the feature;

(c) Retention, which measures the repetitive engagement of active potential customers in the product or feature;

(d) Revenue, which measures the event of being paid by retained customers. At this point, the product/feature, without any doubt, has proven to be viable; and

(e) Referral, which measures the satisfaction of current customers and becomes a new marketing channel by referring new potential customers, which may lead to increased revenue.

Uniqueness. Organizations must find a way to prevent others from duplicating their products. The model proposes the following areas to consider, while this may not prevent duplicating the product, it would make it more difficult to mimic; for example

(a) Personalized Services. The organization must understand its existing customers to serve them better. No two customers are the same [8]. Providing 
personalized services will create a stronger relationship [33], which in turn makes it difficult to imitate.

(b) Barriers to entry. For example, would providing higher quality support help in acquiring new and retaining current customers? Does it sufficiently raise the entry-price of the product space to dissuade similar product offerings?

(c) Inelastic demands. Minimizing sales price deters new products entering the marketplace [11]. This can be realised when the product enters its "growth phase" of customer attraction.

(d) Distributor agreement. How do we build a network-around the product? What are the plans to encourage other products and services to affiliate? Can this affiliation network increase product-switching costs [29]? May this derive new revenue streams?

\section{Implementation Protocol}

The principle objective of this research is to build, implement, and deploy validation-driven development models - that satisfies the components described in section 3.4. The deployment of this process in cooperation with an industrial partner serves as platform to validate or refute these models as viable mechanisms to achieve the exploration of potential products. Thus, it is important to describe some chronological phases on how the models should be implemented and deployed. Even though the phases are overlapping and iterative. We also provide suggestions on how to effectively enhance the process. These phases are described below in more details.

\subsection{Phase I: Analysis of Current State}

Extensive data collection of the current state of the organization and its environment is vital to avoid the pitfalls that can occur during the deployment of a new process [27]. One of the minimum requirements for model-based change includes having two states in place (before/current and the after/future) [17].

In building a model, the current state usually informs, controls, feeds and influences the future state. Comparison between the current and future state enables a transition plan to be developed.

The current state offers a snapshot of the organization's assets, policies and procedures; this assists in developing different models. This also ensures that the deployment occurs efficiently and prevents negative impacts [6].

A successful adoption of the validation-driven model places demands on the organization - right deployment team, adequate deployment strategy and defining the required expertise in order to overcome the current organizational challenge. To establish current state, an analysis should be undertaken to determine the organization's internal abilities and downsides as well as external environment and risks.

A well-established analysis method is SWOT analysis [32, 14], which can be employed to analyze both: 1) the internal factors by analyzing the organizations strengthens and weaknesses, and 2) the external factors by analyzing the opportunities, and threats that the organization may encounter.

\subsection{Phase II: Gap Analysis}

The gap analysis helps to identify the needed improvement projects to transform the organization from its current state as compared to the desired potential performance or future state. Gap analysis is undertaken on three levels including business, product and process levels [6]:

(a) Business level analysis, involves the comparison of the organization's performance to other competitors in the industry. This is achieved by means of benchmarking.

(b) Process level analysis, includes assessment of the optimally performing processes by evaluating the cost, quality and cycle time.

(c) The product level analysis, involves establishing the lack of necessary features, capabilities, and quality that are essential for a competitive product [34].

The modeling of future state plays an important role in identifying the gaps on how the model will be implemented. After identifying the gap, a strategic roadmap is developed where efforts are then focused on the changes required to eliminate the gap between the desired future and the current state [9].

\subsection{Phase III: Modeling Future State}

Frank [12] indicates that while taking into account current organizational readiness, future-state recommendations should be based upon the stakeholders proposed process, the documentation of how the future state will look like, additional process controls and measures, and an outline roadmap for moving the current process to the desired future state.

This gap analysis fully captures relevant information that would influence the future evolution of the model. Gap analysis derives the development of the transition plan; basically, the plan considers three main objectives:

(1) successful implementation of the proposed validation model's components; 
(2) successful adoption of the experiment's characteristics; and

(3) stabilizing and sustaining the performance of the validation-driven model.

The first two objectives were achieved through training, workshops and on-site mentoring. For example, the cost component of the validation model is based around activity-based costing, which means that organizations should convert the indirect cost into direct cost and add it to the overall cost of the experiment, while keeping the cost of the experiment as low as possible, which conform to the characteristic "Support safe-to-fail examination".

Another component is forming interactions with the customers. Interactions can be undertaken through social media from which prototypes of the customer's various needs can be identified and executed accordingly. Social media has grown to be one of the easiest and most convenient means of meeting prospective customers. This supports customers and validation-focused team interactions by establishing a regular, long-term, communication mechanism [7].

Likewise cost and interactions, introducing the concepts, practices, and tools such as MVP, AARRR, Kanban, and horizontal-segmentation amongst others should be facilitated through visiting organized conferences, periodical individual and group meetings platforms.

The third objective should be part of the organization's long-term plan, first, the performance should be assessed, and the model should be refined until it is further stabilized and sustained as described in the next section.

\subsection{Phase IV: Assessment and Refinement}

During the assessment process, an assessor should maintain flexibility and agility of the validation-driven model, whereas during the refinement process, they should ensure dependability despite changes. The assessment process is concerned with evaluating the performance with regard to the aforementioned model's components and characteristics. While the refinement process is focused on enhancing the overall performance of the model.

Hence, the assessment should be conducted at a micro level for each of the deployed components of the proposed validation model. For the sake of brevity, we only discuss some examples of this assessment:

Safe-to-fail experiments, the ability to allow "non-successful" ideas to fail in small and tolerable ways. These experiments generate observable benefits that can be amplified or adopted by the customers. The customers will then offer beneficial feedback through satisfaction surveys and/or interviews about the extent to which safe-to-fail experiments promoted visibility of emergent possibilities during the design process.

Are the AARRR metrics sufficient? Their intended purpose is measured against the predetermined business growth objectives through the engines of growth model [25]. The metrics are then evaluated to check whether they provide enough data about the clients, retention of customers, and revenue generation?

Interactions with customers. In order to assess the worth of the employed communication channels, free analytics tools can be utilized [19]. For example, Google Analytics provides means to measure the impact of these channels on the achievement of the predefined goals. Examples of these goals include predetermined targeted number of activated customers, or reduction of customer acquisition cost.

Customer-segmentation. Appropriate segmentation can be assessed by evaluating the effectiveness of vertical and horizontal-segmentations [22] in defining the customer's challenges; The evaluation criteria may include: homogeneity (the extent to which consumers within a segment value similar features), heterogeneity (each segment of customers should be unique), substantiality (market segment being large enough it terms of profitability and sales), and response (extent to which market segments react to communication).

The outcome of the assessment process is then used to inform the refinement process. The model should be iteratively refined based around the actual needs of the customers and the identified weakness that emerge from the use of the proposed validation model. This analysis will help with identifying pitfalls and weaknesses. Subsequently, the models may either run in parallel with additional models that overcome the weaknesses, or be replaced entirely by a wellestablished model(s) that has proven to be successful within the organization's context.

The resulting new models will go through further assessments and refinements until the overarching validation-driven model becomes stabilized. The refinement process should, for example, consider:

(a) If the safe-to-fail experiments require refinement based on the information gathered from the customers, more efficient and effective experiments will then be developed to make emergent issues of design to be more visible so that non-beneficial ideas fail in a tolerable manner.

(b) If the current communication and interactions do not reach a large customer base, necessary refinement will be undertaken by developing effective channels that will boost more reliable communications. Cost will be a paramount aspect in refining communication channels. Therefore, 
the most cost-effective channels that reach the highest number of "relevant" potential customers should be offered the most consideration.

(c) Customer segmentation could be refined to ensure homogeneity for customers with similar needs; the focus should be on developing a more effective response to the unique customer problems within each segment, while ensuring uniqueness between different segments.

\section{Methodology}

The proposed model has been developed incrementally and gradually using Action Research methods (Easterbrook et al., 2008; Dietrich et al., 2008). The primary investigator has been working with the industrial partner on a daily-basis for extended period of time to understand and craft strategies to assist in implementing the proposed model.

The primary investigator has been in-charge of dayto-day interactions with research participants and has been the designer on many of the details in the current validation model, hence, he was considered normal participant rather than observing participant [17].

In an attempt to limit data misinterpretation, direct and indirect data collection techniques were used to validate the findings (Easterbrook et al., 2008; Dittrich et al., 2008). The direct techniques included surveys and semi-structure interviews, focused groups through brainstorming workshops, mainly observational.

The researchers have also sought expert opinion and feedback to help validating the interpretation of critical findings. The indirect techniques used to collect data had included analysis of tool logs and documentation analysis (Singer et al., 2008), which were obtained through system logs, including for example the tasks distribution system that the industrial partner uses.

\section{Discussion}

The proposed model is composed of set of processes, methods, tools, and techniques that allow software-centric organizations to integrate lean principles in managing the development process and combining it with proven value-driven agile methods to improve the overall performance of the development processes.

Test-Driven Development (TDD) is a proven agile method. TDD's core concept is based around writing test cases prior to developing and refactoring the actual code. Following the same concept, we propose validating the product idea before actually building the product. Although, test-driven methods are mainly for verification and not validation, verification (and validation)-driven processes are believed to provide a mechanism to start evaluating the uncertainty or risks associated with product development. Hence, we propose that:

Successful software product development needs to utilize validation-driven processes early in its lifecycle. These validation-driven processes will eventually give way to verification-driven processes during development.

The proposed model is concerned with the early stages of the product development, and concentrates on the hypothesis that value to the customer should be the principle driver when designing a lifecycle. The key is to develop a working definition of value. We are seeking to define value from "cradle to grave". Hence, we concentrate on learning the context of the product instead of focusing on the delivery of the product!

Interactions with customers to learn more about their needs forms the heart of the validation model. The team should continuously validate with the customer base by VOC methods. Regardless of the methods used, it is important to systematically and continuously validate with the customer base.

It is also important to note that the validationdriven processes are totally different from the verification-driven processes. The former seek to reduce the risks directly associated with customers, i.e. maximise take-up; whereas the later seek to reduce the risk associated with the code base, i.e. minimise defects and omissions. These processes require that verification and validation practitioners to have very different skillsets.

Hence, we propose: Software Development teams should be composed of two sub-teams - one is validation-focused, and one is verification-focused. This implies moving the production process to a crossfunctional team arrangement. This arrangement would then support the new value-oriented goal of the production process.

\section{Related Work}

Several research studies have attempted to integrate a number of the methods, techniques and approaches discussed in section 3.4, these studies, however, looked at each method at a micro-level. It is our strong believe that research studies aimed at exploring ways to integrate several proven approaches at a macro-level, have a greater success rate with realizing the desired results. 
Eric Ries [25] for example, portrayed the lean concepts as a methodology and management system. In his book "The Lean Startup", the approach is claimed to help entrepreneurs in providing an innovative process to inform the investment decisions and increase the success rate of establishing a new business. The approach is built around assessing the interests of a business idea with the customer base prior building the actual business. The Lean Startup suggests the use of learning loops, MVP, and experiments.

We have established the validation model based around these concepts, however, we have also considered the social and behavioral aspects of the organization and the team members. Organization behavior and culture can never be disregarded when developing such model. The proposed validation model tries to combine, lean, agile, and value-driven design into one model.

Bolchini et al [5] developed a novel approach to value-driven design in the context of web requirements engineering, the scholars claim that the approach links business value to user needs. The approach has been claimed to be a multidisciplinary framework combining web requirements engineering, to brand design and marketing, with value-driven design.

Value driven approaches, however, can never exist by themselves. It must demonstrate strong ties to customer involvement. The proposed validation model utilizes VOC and embeds it within every step and action throughout the product development process.

Shen et al. [30] propose a model that integrates VOC, to quality function deployment (QFD), with Kano model analysis (questionnaire approach). Despite the fact that this model considered the involvement of the customer, it has major drawbacks and flaws. For example, the model suggests, as cited from [30, pp 94]:

The project team must decide who may have interest in this product ... team members may have varied ideas of who constitute potential customers.

In order to provide a systematic and innovative model, the process should consider identifying the right customer base. We believe that Shen's model shifts the defects in the process from assuming the customer needs to assuming who may constitute the customer base! The proposed validation model overcomes this weakness by systematically identifying the relevant customer segments to elicit their feedbacks.

\section{Conclusions}

Competition in the software engineering-centric market is becoming increasingly intense. This has led organizations seeking new ways to achieve sustainable advantages. The proposed validation-driven development model has been designed based on combining proven industrial methods to realize the sustainable competitive advantage by continuously improving the development process.

Software product development has been shifting from feature-driven, to behavioural -driven, until the trend has become the value-driven development, which promotes maximizing the product value rather than meeting specific product functional or performance requirements.

The proposed validation model concentrates on the early stages of the product development and attempts to overcome the pitfalls and weaknesses that have been reported in the literature in regard with the dominant product development lifecycles.

This study attempts to integrate Value-Driven approaches, Agile methods, and Lean principles and best practice into one model. The ultimate goal has been to deliver value to the end customers. Value, however, should be determined from the customers' perspective.

The definition of value may differ from one customer segment to another, hence, the successful product should consider multiple value propositions to satisfy the different customer tastes. The value of a software product is not limited to its code-base, the value should be present in every activity within the development process.

Product development lifecycle includes supporting functions, such as marketing, accounting, and customer service, defining value in these areas have been often overlooked. The proposed validation-model attempts to consider value form "cradle to grave". The cost of marketing may be argued to be a non-value added expense (from the customer perspective), however, the marketing helps in branding the product, which is definitely appreciated by the customer base. The proposed model provides a means to validate value delivered to the customer-base for every expense occurred during the product development process.

While no efforts have been spared to generalize the model and make it suitable for deployment in diverse settings, care, however, should be taken when implementing this model, the deployment protocol may not fit in non-typical environments. For example, an organization is required to restructure the development team. The validation model touches the organization behaviour and structure, not only the development process, hence an organization may need to reframe some methods to fit its unique context.

The model can be very useful and more suitable for organizations that are considering restructuring their teams and re-engineering their development processes. 


\section{References}

[1] Al-Baik, Osama, and James Miller. "Kaizen Cookbook: The Success Recipe for Continuous Learning and Improvements." In 2016 49th Hawaii International Conference on System Sciences (HICSS), pp. 5388-5397. IEEE, 2016.

[2] Al-Baik, Osama, and James Miller. "The kanban approach, between agility and leanness: a systematic review." Empirical Software Engineering 20.6 (2015): 1861-1897.

[3] Baker, Ronald J. Pricing on purpose: Creating and capturing value. John Wiley \& Sons, 2010.

[4] Besterfield Dale, H. Quality control. Pearson Education.

[5] Bolchini, Davide, Franca Garzotto, and Paolo Paolini. "Value-driven design for infosuasive web applications." Proceedings of the 17th international conference on World Wide Web. ACM, 2008.

[6] Brörkens, Mark, and Ömer Gürsoy. "Managing and understanding complex systems using traceability and open source software." INCOSE International Symposium. Vol. 22. No. 1. 2012.

[7] Chou, Paul B., Edna Grossman, Dimitrios Gunopulos, and Pasumarti V. Kamesam. "Prospective customer selection using customer and market reference data." U.S Patent No. 6,061,658. 9 May 2000.

[8] Croll, Alistair, and Benjamin Yoskovitz. Lean analytics: use data to build a better startup faster. " O'Reilly Media, Inc.", 2013.

[9] Curtis, Bill, Herb Krasner, and Neil Iscoe. "A field study of the software design process for large systems." Communications of the ACM 31.11 (1988): $1268-1287$.

[10] E. Juergens, M. Feilkas, M. Herrmannsdoerfer, F. Deissenboeck, R. Vaas, and K. Prommer, "Feature profiling for evolving systems," 19th IEEE International Conference on Program Comprehension, 2011.

[11] Ellingsen, Tore. "Price signals quality: The case of perfectly inelastic demand."International Journal of Industrial Organization 16.1 (1997): 43-61.

[12] Frank, Ulrich, Oscar Pastor, Pericles Loucopoulos, and Ilias Petrounias. The Practice of Enterprise Modeling: 7th IFIP WG 8.1 Working Conference, PoEM 2014, Manchester, UK, November 12-13, 2014, Proceedings. Vol. 197. Springer, 2014.

[13] Guide.agilealliance.org,. 'Guide To Agile Practices', 2013. Web. 13 May 2016.

[14] Houben, Ghislain, Kwan Lenie, and Koen Vanhoof. "A knowledge-based SWOT-analysis system as an instrument for strategic planning in small and medium sized enterprises." Decision support systems 26.2 (1999): 125-135.

[15] Johnson, Jim. "ROI, It's your job." Published Keynote Third International Conference on Extreme Programming, Alghero, Italy. 2002.

[16] Kohavi, Ron. "A study of cross-validation and bootstrap for accuracy estimation and model selection." Ijcai. Vol. 14. No. 2. 1995

[17] Li, Shuai, Frank Singhoff, Stéphane Rubini, and Bourdellès Michel. "Applicability of real-time schedulability analysis on a software radio protocol." ACM SIGAda Ada Letters. Vol. 32. No. 3. ACM, 2012.

[18] Love, Gregory, and Geoffrey Back. "Model verification and validation for rapidly developed simulation models: Balancing cost and theory." Proceedings of the 18th international conference of the system dynamics society. 2000.

[19] Maurya, Ash. Running lean: iterate from plan A to a plan that works. " O'Reilly Media, Inc.", 2012.

[20] McClure, Dave. 'Startup Metrics For Pirates'. Slideshare.net, 2007. Web 13 May 2016

[21] Mellor, Stephen J., Kendall Scott, Axel Uhl, and Dirk Weise. "Model-driven architecture." In International Conference on Object-Oriented Information Systems, pp. 290-297. Springer Berlin Heidelberg, 2002.

[22] Mentzer, John T., Matthew B. Myers, and Mee-Shew Cheung. "Global market segmentation for logistics services." Industrial Marketing Management 3(2004): $15-20$.

[23] Müller, Roland M., and Katja Thoring. "Design thinking vs. lean startup: A comparison of two user-driven innovation strategies." LEADING THROUGH DESIGN (2012): 151.

[24] PwC, A Nation Of Innovators: 2015 Canadian Emerging Companies' Survey. 2015. Web. 13 May 2016.

[25] Ries, Eric. The lean startup: How today's entrepreneurs use continuous innovation to create radically successful businesses. Random House LLC, 2011.

[26] Robinson, Ross. "Ports as elements in value-driven chain systems: the new paradigm." Maritime Policy \& Management 29.3 (2002): 241-255.

[27] Sasankar, Ashish B., and Vinay Chavan. "SWOT Analysis of Software Development Process Models." IJCSI International Journal of Computer Sciences Issues 8.3 (2011).

[28] Senge, Peter M. The fifth discipline: The art and practice of the learning organization. Broadway Business, 2006.

[29] Shapiro, Carl, Hal R. Varian, and W. E. Becker. "Information rules: a strategic guide to the network economy." JOURNAL OF ECONOMIC EDUCATION 30 (1999): 189-190.

[30] Shen, Xiao-Xiang, Kay C. Tan, and Mien Xie. "An integrated approach to innovative product development using Kano's model and QFD." European journal of innovation management 3.2 (2000): 91-99.

[31] Turney, Peter BB. "Activity based costing." Hall, Johnson and Turney (ed.): "Measuring Up, Charting Pathway to Manufacturing Excellence", Business One Irwin, Illinois (1990).

[32] Valentin, K. "SWOT analysis fromresource-based view" Journal of Marketing theory and Practice (2001): 54-69.

[33] Van Der Riet, Ramon. "Marketing communication and transaction/distribution services platform for building and managing personalized customer relationships." U.S. Patent No. 7,917,388. 29 Mar. 2011.

[34] Xu, Lida, Chengen Wang, Xiaochuan Luo, and Zhongzhi Shi. "Integrating knowledge management and ERP in enterprise information systems." Systems Research and Behavioral Science 23.2 (2006): 147-156. 